\title{
AVALANCHE DYNAMICS IN DISORDERED SYSTEMS
}

\author{
ALBERTO ROSSO (*) \\ Nota presentata dal m.e. Attilio Rigamonti
}

(Adunanza del 20 dicembre 2012)

Sunto. Descriviamo la dinamica complessa di un'interfaccia elastica che si muove in un mezzo disordinato e in presenza di una forza esterna. Quando il valore della forza esterna è abbastanza piccolo, il moto dell'interfaccia è lento e intermittente. Due diversi regimi sono osservati. Un primo regime detto di creep si presenta per valori della forza esterna molto piccoli. Qui il moto è possibile solo per attivazione termica e l'interfaccia appare per lo più bloccata in profondi stati metastabili separati da grandi barriere energetiche. Un secondo regime detto di depinning è caratterizzato dalla presenza di moto anche a temperatura nulla grazie ad una dinamica che procede attraverso riorganizzazioni di grandi porzioni dell'interfaccia dette valanghe. I nostri risultati gettano nuova luce sulla transizione tra i due regimi e sulla statistica delle valanghe.

$$
* * *
$$

AвSTRACT. We describe the complex dynamics of an interface (like a magnetic domain wall) on a disordered landscape. In presence of a small external drive (i.e. an external magnetic filed) the motion of the interface is slow and jerky. Two different regimes can be distinguished: the thermally activated regime where the interface spend most of the time in deep metastable states separated by large barriers (creep regime) and the avalanche regime where motion persists also at zero temperature, but it involves collective re-organizations (depinning). Our results shed light on the interplay between these two regimes and on the avalanche statistics.

${ }^{(*)}$ Dipartimento di Fisica, Laboratoire Physique Théorique et Modèles Statistiques (UMR CNRS 8626), Université Paris-Sud, Orsay Cedex, France.

E-mail: alberto.rosso@u-psud.fr 


\section{INTRODUCTION}

The growth of interfaces in a disordered medium is a longstanding problem with important applications for a host of experimental systems. Such problems can be split into two broad categories: (i) propagating interfaces such as magnetic domain walls [1, 2], contact line in wetting [3], and crack propagation $[4,5,40]$; (ii) periodic systems such as vortex lattices [7], and charge density waves [8]. In all these systems the basic physical ingredients are identical: the elastic forces tend to keep the structure ordered (flat for an interface and periodic for lattices), whereas the impurities locally promote the wandering (see Fig. 1). From the competition between disorder and elasticity emerges a complex energy landscape with many metastable states. This results in glassy properties such as hysteresis and history dependence of the static configuration.

If we consider an interface at equilibrium with the random environment, scaling arguments due to Larkin [9] show that below four (internal) dimensions, displacements grow unboundedly with the distance, resulting in rough interfaces even at zero temperature. The roughness exponent, $\zeta_{\text {eq }}$, characterise the spatial wandering of the interface and depends only on the correlation of the disorder, the dimension of the interface, and the nature of the elastic forces.

The competition between disorder and elasticity manifests also in the dynamics of such systems. Among the dynamical properties, the response of the system to an external force $f$ is specially crucial, both from a theoretical point of view and respect to measurements. Indeed, in most systems the velocity $v$ versus force $f$ characteristics is directly measurable and is simply linked to the transport properties (velocity-applied magnetic field for magnetic domain walls, crack velocity-elastic energy release rate $G, \ldots)$. Different dynamical regimes can be observed as shown in Fig. 2.

The Creep regime. It is natural to expect that under the action of a small applied force, at zero temperature, the system remains pinned and only moves until it locks on a local minimum of the tilted energy landscape. In this regime, the system is expected to move through thermal activation. What is the nature of this motion and what is the velocity? The first model of such a motion, called TAFF (Thermally Assisted Flux Flow) model, found a linear response [10]. The idea is to consider that a 


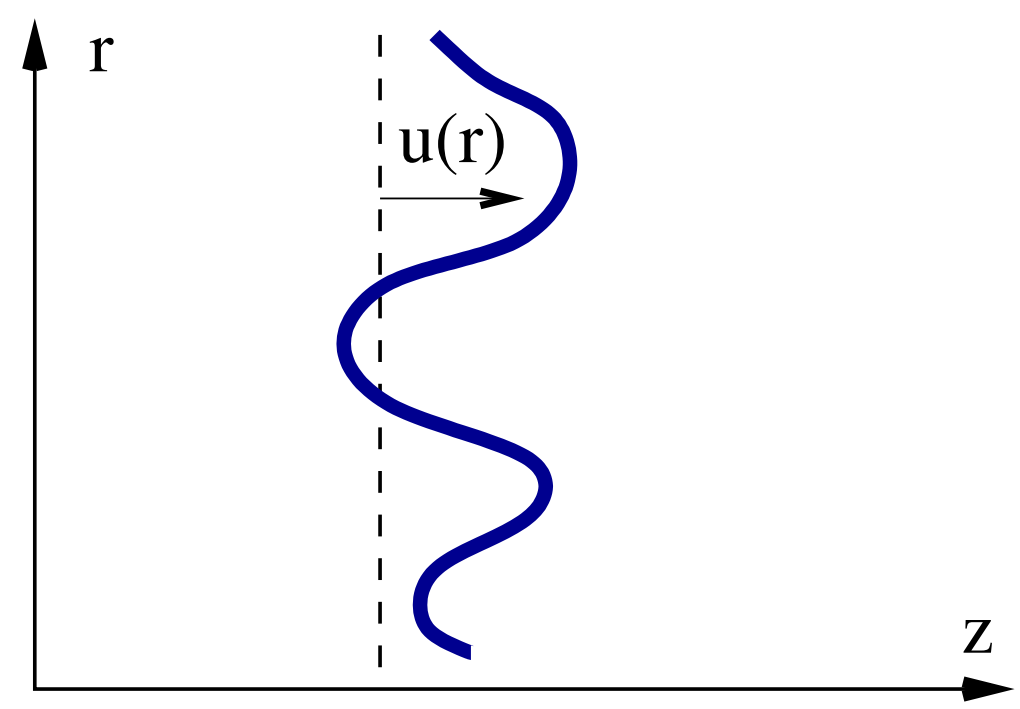

Fig. 1 - A one dimensional interface (domain wall) living in a two dimensional space (film). The position of the interface is determined (provided there are no overhangs or bubbles) by the displacement $u$ from a flat configuration. The roughness of the interface is characterised by the exponent $\zeta,(u(r)-u(0)) \sim r^{\zeta}$.

blob of pinned material has to move in an energy landscape with characteristic barriers $U_{b}$ as shown in Fig. 3. The external force $f$ tilts the energy landscape making forward motion possible. The barriers are overcome by thermal activation (hence the name: Thermally Assisted Flux Flow) with an Arrhenius law. If the minima are separated by a distance $a$ the velocity is

$$
v \propto e^{-\left(U_{b}-f a / 2\right) / T}-e^{-\left(U_{b}+f a / 2\right) / T} \simeq e^{-U_{b} / T} f
$$

The response is thus linear, but exponentially small. However, this argument is grossly inadequate for a glassy system. The reason is easy to understand if one remembers that the static system is in a vitreous state. In such states a characteristic barrier $U_{b}$ does not exist, since barriers are expected to diverge as one gets closer to the ground state of the system. The TAFF formula is then valid in systems where the glassy aspect is somehow killed and the barriers do saturate. This could be the case for 


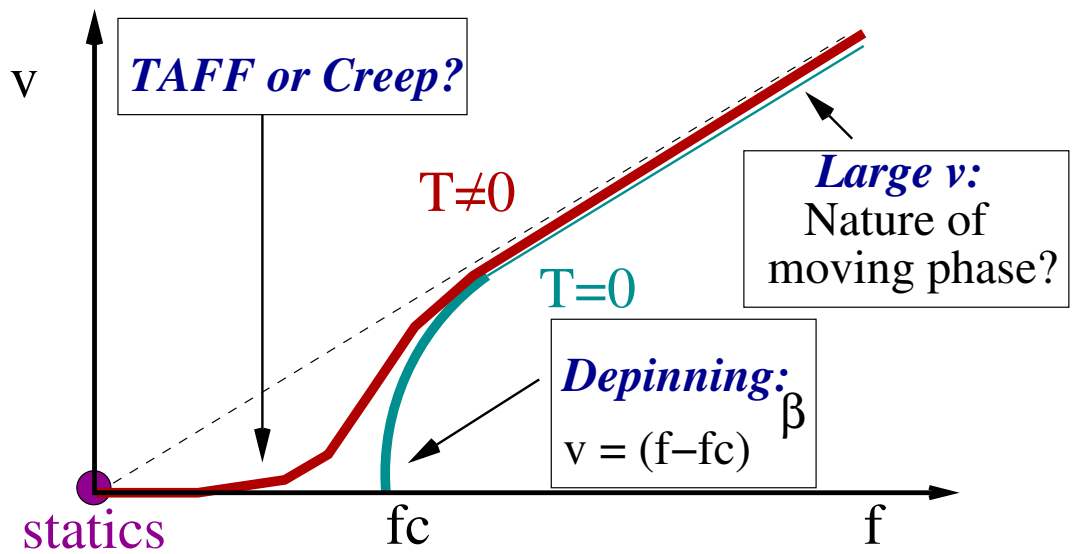

Fig. 2 - The velocity $v$ - $f$ characteristic of a disordered elastic system. At zero temperature the system stays pinned until a critical force $f_{c}$ is reached. At finite temperature a motion can occur even for forces below threshold $f<f_{c}$ since the barriers to motion can always be passed by thermal activation.

example for a finite size interface. When the glassy nature of the system persists up to arbitrarily large length scales the motion is actually dominated by barriers which diverge as the drive $f$ goes to zero. To evaluate the size of these barriers, scaling arguments rely on strong assumptions such the use of statics properties to describe an out of equilibrium system [11]. This phenomenological analysis, confirmed by a functional renormalization group calculation [12], leads to the so called creep formula

$$
v \sim \exp \left(-C f^{-\mu_{\mathrm{eq}}} / T\right)
$$

where $\mu_{\mathrm{eq}}$ is an equilibrium critical exponent. This formula has recently been verified by experiments on magnetic domain walls [1,2].

The depinning regime At larger drive, the system follows the force $f$, even at zero temperature, and acquires a non-zero asymptotic velocity $v$. What is the depinning threshold $f_{c}$ below which the interface is immobile and above which a steady-state motion sets in? An estimate of $f_{c}$ can be obtained via scaling arguments [9] or computed numerically 


\section{energy landscape}

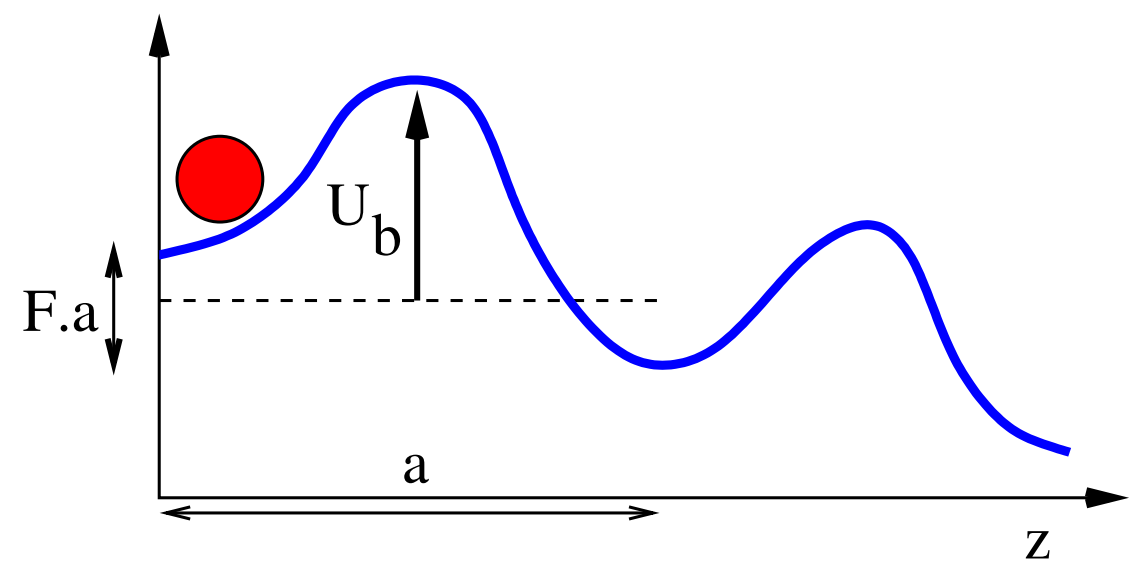

Fig. 3 - Left: In the Thermally Assisted Flux Flow (TAFF) [10] a region of pinned material is considered as a particle moving in an energy landscape characterized by characteristic barriers $U_{b}$. This leads to a small but linear response.

by an exact algorithm [13]. Close to $f_{c}$ the driven dynamics proceeds by discontinuous collective rearrangements called avalanches. The size of these rearrangements grows as we approach to the threshold and, at the transition $f=f_{c}$, the avalanche statistics is expected to be scale free [14]. In analogy with equilibrium critical phenomena, the scaling behaviour is characterised by universal critical exponents, such as the correlation length exponent, $\nu_{\text {dep }}$, related to the linear extension of the avalanches $\left(\xi(f) \sim\left|f-f_{c}\right|^{-\nu_{\text {dep }}}\right)$ or the velocity exponent, $\beta_{\text {dep }}$, defined as $v \sim\left(f-f_{c}\right)^{\beta_{\text {dep }}}$. The value of these exponents has been determined by analytical techniques such as the functional renormalization group $[15,16]$ and by numerical approaches [17].

In this paper we discuss the recent advanced in our comprehension of the dynamical phase diagram shown in Fig. 2. In particular, in Section 2 we account for a unified description of the dynamics below $f_{c}$ and in particular we explain how to match the creep regime with the depinning transition $[18,19]$. In Section 3 we focus on the depinning transition and characterize the critical force and the avalanche statistics. 

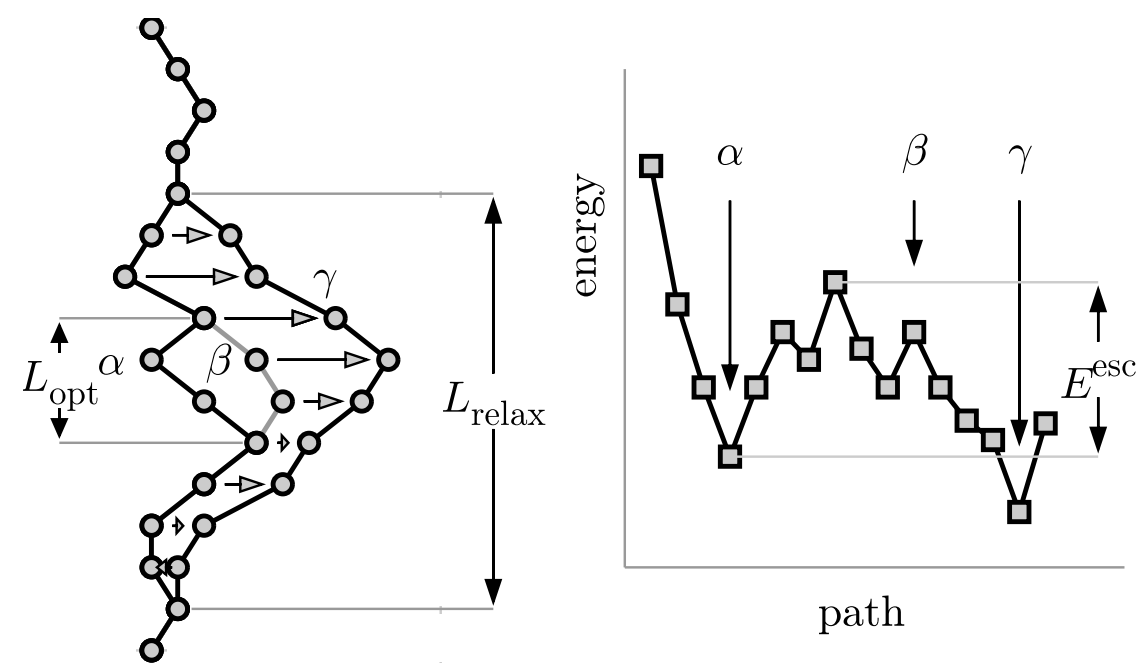

Fig. 4 - Low temperature dynamics of the driven elastic string below the depinning threshold: the optimal path to escape from a given metastable configuration $\alpha$ pass through a saddle configuration $\beta$ that can relax deterministically to the next metastable configuration $\gamma$ with $E_{\gamma}<E_{\alpha}$.

\section{DEPINNING VS CREEP: THE DYNAMICAL PHASE DIAGRAM BELOW THRESHOLD}

\subsection{Creep formula: a divergent length scale when $f \rightarrow 0$}

The slow dynamics of the interface for $f \rightarrow 0$, so-called creep, is controlled by thermally activated jumps of correlated regions over the pinning energy barriers separating different metastable states. Scaling arguments are based on the assumption that the dynamical barriers of this out-of-equilibrium system can be determined purely from the statics.

In general three different dynamical steps can be isolated (see Fig. 4): (i) Starting from a deep metastable state, $\alpha$, the interface explores the neighborhoods jumping up and down over energy barriers. (ii) This continues until a saddle configuration $\beta$ is found. (iii) Finally the configuration $\beta$ can relax deterministically to a new and deeper metastable configuration $\gamma$. Configurations $\alpha$ and $\beta$ differ on a length $L_{\text {opt }}$ while $\alpha$ and $\gamma$ differ on a length $L_{\text {relax }}$. 


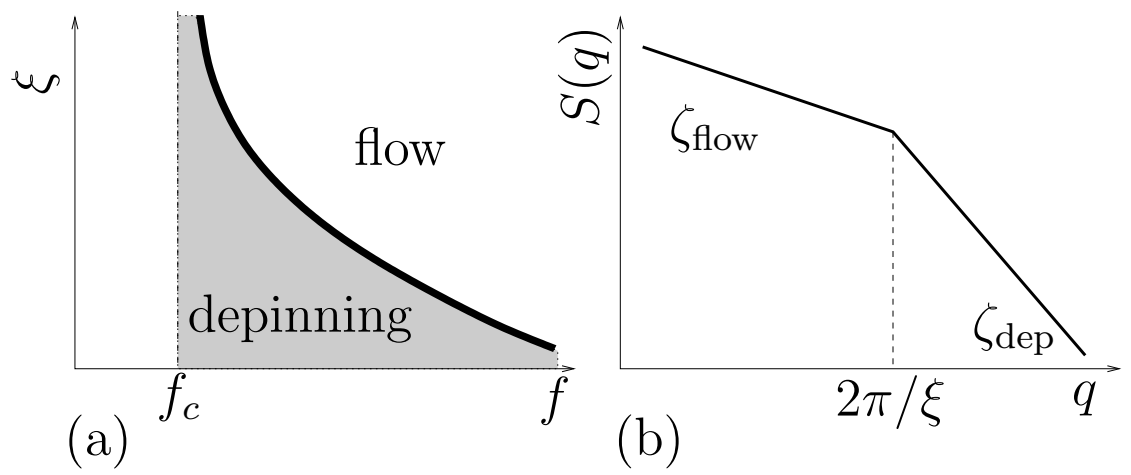

Fig. 5 - (a) Zero-temperature dynamical phase diagram. (b) Schematic structure factor for $f>f_{c}$. The correlation length $\xi$ fixes the crossover between depinning and fast flow. As an example, for a one dimensional interface with short range disorder we have $\zeta_{\text {dep }} \sim 1.25$ and $\zeta_{\text {flow }}=0.5$.

The size of the optimal excitation $L_{\mathrm{opt}}$ is obtained by the balance of the gain in energy of being pinned in a deep metastable state, $E_{\text {pinned }}(L)$, with the gain in energy of moving the interface forward, $E_{\text {depinned }} \sim$ $f \cdot\left(u_{\beta}(L)-u_{\alpha}(L)\right)$. At equilibrium we have that $u_{\beta}(L)-u_{\alpha}(L) \sim L^{\zeta_{e q}}$ and that $E_{\text {pinned }}(L) \sim L^{\vartheta}$, with $\vartheta=2 \zeta_{e q}-1$ (this exact relation is due to the statistical tilt symmetry [20]). The balance gives that

$$
L_{o p t} \sim f^{-\nu_{e q}} \quad \nu_{e q}=\frac{1}{2-\zeta_{e q}},
$$

which means that $L_{\text {opt }}$ diverges when $f \rightarrow 0$.

Moreover, numerical simulations give a clear evidence that the energy landscape is characterized by a unique energy scale, and that the energy difference between neighbor metastable states is equal to the energy barrier separating them [21]. Thus, we expect that the barrier that the interface has to overcome to escape from the state $\alpha$ grows as $L_{o p t}^{\vartheta} \sim$ $f^{-\frac{2 \zeta_{e q}-1}{2-\zeta_{e q}}}$. Using the Arrhenius activation, we recover the creep formula Eq. (2) with $\mu=\left(2 \zeta_{e q}-1\right) /\left(2-\zeta_{e q}\right)$. 


\subsection{The depinning transition: a divergent length scale when $f \rightarrow f_{c}$}

Above the critical force and at zero temperature the analogy of the depinning transition with critical phenomena is well understood [14]: As in a thermodynamic second-order phase transition, the connected twopoint correlation function of the order parameter is characterized by a correlation length which diverges at the critical point. The steady-state velocity is the order parameter of the depinning transition and its twopoint correlation function,

$$
\overline{\langle(v(x, t)-v)(v(0, t)-v)\rangle} \sim e^{-|x| / \xi},
$$

indeed diverges for $f \rightarrow f_{c}^{+}$. In practice, this correlation function is not easily accessible because the steady-state velocities can be obtained only after a long-time integration of the equation of motion [22].

The correlation length $\xi$ separates two length scales in the manifold (see Fig. 5a): on scales smaller than $\xi$, the geometry, i. e. the roughness, of the interface is characterized by depinning exponents (the exponents of the critical phase in the language of magnetic transitions). In contrast, on length scales larger than $\xi$, the interface is governed by the fast flow exponents (analogous to the exponents of the ferromagnetic ordered phase). The length $\xi$ can be measured through the power spectrum or structure factor [23],

$$
\begin{aligned}
S(q) & =\overline{\left\langle\left|\frac{1}{L^{1 / 2}} \int d x u(x, t) e^{-i q x}\right|^{2}\right\rangle} \\
& =\int d x e^{-i q x} \overline{\langle u(x, t) u(0, t)\rangle}
\end{aligned}
$$

where the second equality makes use of spatial translation invariance. For inverse lengths $q$ belonging to a self-affine regime with a single roughness exponent $\zeta$, the structure factor takes the form

$$
S(q) \sim q^{-(1+2 \zeta)} .
$$

The crossover between the depinning and the fast-flow regimes can be conveniently extracted from the change of slope of $S(q)$ (see Fig. 5b). 


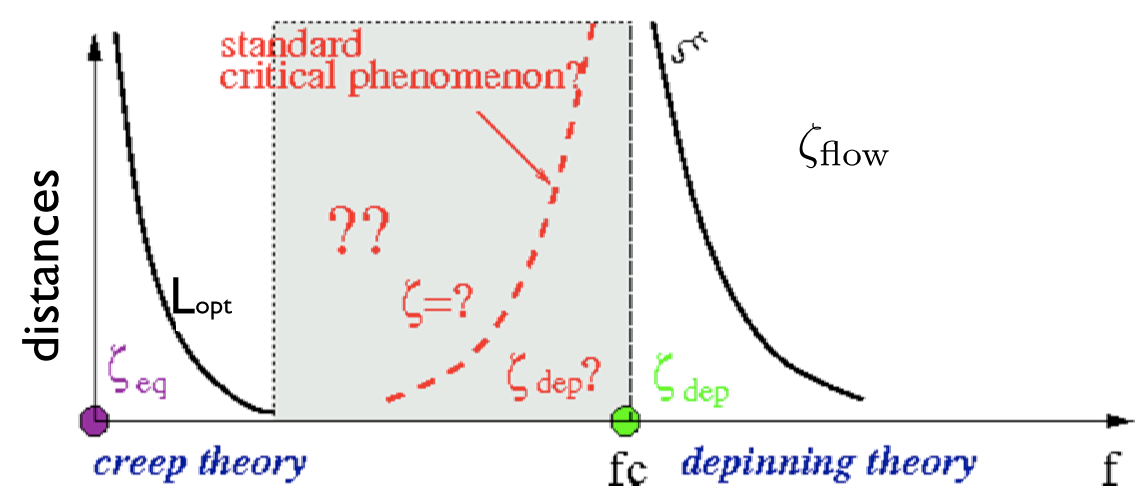

Fig. 6 - Dynamical phase diagram, the geometry of the interface in the steady state regime. Below threshold we expect two divergent correlation length: the activation size $L_{\text {opt }}(f)$ which diverges as $f \rightarrow 0$ and the distance from consecutive metastable states $\xi(f)$ which diverges as $f \rightarrow f_{c}$. Below the scale $L_{\text {opt }}$ we expect that the system is at equilibrium, below the scale $\xi(f)$ the system is at the depinning critical point. How behaves the system at larger length scales?

Below the depinning threshold $f_{c}$, at zero temperature, the manifold is permanently pinned. The interpretation of depinning as a critical phenomenon suggests that a similar diverging length scale should also exists below $f_{c}$. Indeed, in standard critical phenomena, for length scales smaller than the correlation length the system is critical, and crosses over beyond this correlation length to the broken symmetry phase on one side of the transition and to the symmetric phase on the other side. An important question is thus whether an equivalent correlation length is observable in the limit $f \rightarrow f_{c}^{-}$.

A classical protocol to study the depinning transition below threshold is defined as follows: we prepare the system with an initial non-steady state configuration, for example the flat configuration, and then we apply an external force $f<f_{c}$. The interface moves forward and re-organize on a length scale $\xi(f)$ which diverges when $f \rightarrow f_{c}$. We will see in Section 3 that this collective re-organization is a manifestation of the avalanche dynamics in these systems. The geometry of the line is governed by the scale $\xi$ : on scales smaller than $\xi$, the roughness of the interface is characterized by depinning exponents. In contrast, on length scales larger than $\xi$, the interface is reminiscent of the non-steady state 
initial condition (in our example it will be flat). This means that, below threshold, a correlation length $\xi(f)$ exists in the transient regime. It is natural to ask if it is possible to study the steady state regime below threshold. If yes, how behaves the system at large length scales? In analogy with what happens above threshold one should expect that, below threshold, the large scale behavior is described by equilibrium exponents of the $f=0$ fixed point. However, as shown in Fig. 6 this does not match with the creep scaling arguments, for which $f=0$ is unstable fixed point. In others words, for the creep scenario, when $f>0$, the interface is at equilibrium only at length scales shorter than $L_{\mathrm{opt}}(f)$.

\subsection{Dynamical phase diagram for $T \rightarrow 0^{+}$}

In $[18,19]$ we studied the steady state regime of an interface in the limit of vanishing temperature. The temperature allows to forget the initial condition and reach the stationary state. A direct numerical study of the motion of such systems at low temperature is very difficult for forces below the depinning force. Indeed, in that case, the motion takes place by thermal activation over barriers leading to extremely long activation times. In this regime numerical techniques such as the molecular dynamics are inefficient. For this reason we introduced a novel numerical method which allows to follow the motion of an interface at finite temperature, without running into the above-mentioned difficulties. This method directly implement the dynamics of Fig. 4. We have proved theorems that assure that this dynamics is the correct low temperature dynamics for a finite interface.

Our results are summarized in Fig. 7. The crossover length, $L_{\text {opt }}$, separates two roughness regimes: On length scales smaller than $L_{\text {opt }}$ the roughness of the interface is described by the equilibrium exponent $\zeta_{e q}$, corresponding to the paramagnetic phase in the language of magnetic transitions. For distances bigger than $L_{o p t}$, the roughness is described by depinning exponents. This is at variance with standard critical phenomenon, where the critical phase at large length scales appears only at the critical point. Increasing the external force, Lopt decreases and when the depinning threshold is reached $\left(f \rightarrow f_{c}^{-}\right), L_{\text {opt }}$ becomes of the order of the lattice space. The depinning divergent length scale $\xi(f)$ can be identified with the characteristic length $L_{\text {relax }}$ of the creep regime. 


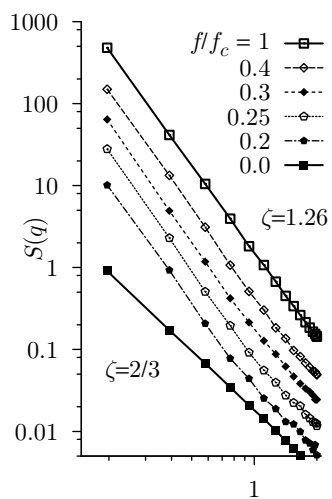

$2 \sin (q / 2)$

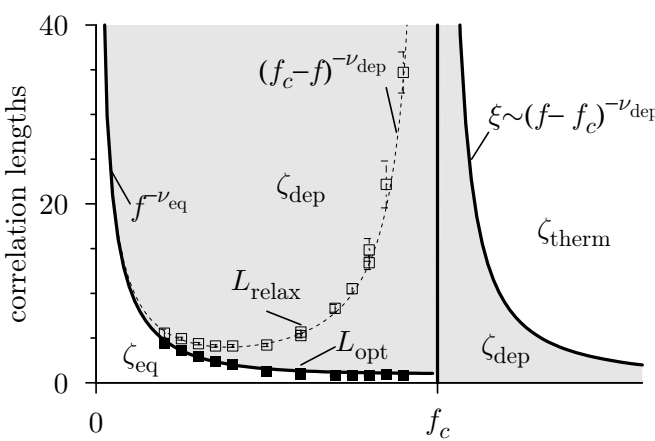

driving force $f$

Fig. 7 - Results: (a) Steady state structure factor of the line in the $T \rightarrow 0$ limit, averaged on 1000 samples. (a) $S(q)$ for $L=32, M=92$ and different forces (curves are shifted for clarity). (b) Summary: The steady state properties of the elastic string at $T \rightarrow 0$ are determined by $L_{\text {opt }}$ (filled symbols) and by $\xi$. They separate regions characterized by the equilibrium exponent, the depinning exponent (gray region), and the thermal or flow one. The divergent length $L_{\text {relax }}$ (open symbols) is associated only with transient dynamics. Lines are guides to the eye.

In the creep $L_{\text {relax }} \sim L_{\text {opt }}$, at the depinning transition $L_{\text {relax }}$ diverges, while $L_{\text {opt }}$ remains finite.

Our algorithm cannot access very small forces, but our results are compatible with predictions in the creep regime. In particular, the functional renormalization group [12] predicts that on scales below $L_{o p t}$ the system is in equilibrium and that scales larger than $L_{\text {opt }}$ are characterized by deterministic forward motion.

\section{AVALANCHE DYNAMiCS}

The driven dynamics of an elastic interface proceeds by discontinuous collective rearrangements. We have introduced two different type of rearrangements: the activated rearrangements of scale $L_{o p t}$ and responsible of the creep regime, and the deterministic re-arrangements of scale $L_{\text {relax }}$ and called avalanches. Here we focus on avalanches.

Avalanches are observed in a number of systems. Some examples are the Barkhausen noise in magnets [24, 25], dislocations and crack 


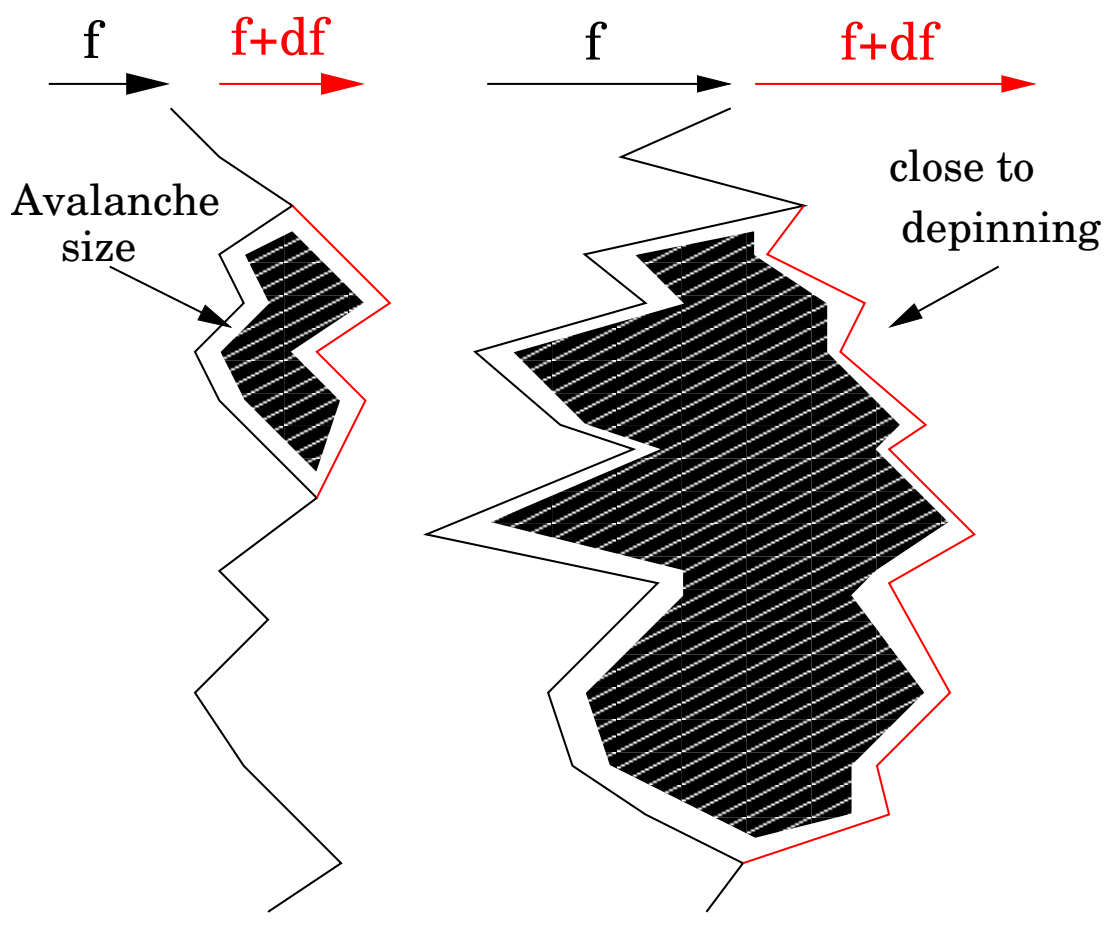

Fig. 8 - Consider an interface pinned in a metastable state in the presence of a force $f$. If we apply an additional infinitesimal force $f \rightarrow f+\delta f$ the metastable state can become unstable and re-organize on very large length scale. The characteristic (maximal) size of the avalanche is called $S_{m}$ and diverges when we approach the depinning transition.

propagation [26, 27], and stick-slip motion of tectonic plates (responsible for earthquakes) [28, 29]. Avalanches have also been studied in models without quenched substrate disorder, such as sandpile models and granular matter [30, 31].

Important characteristics of avalanche motion are its scale invariance, self-organized criticality, and a broad distribution $P(S) \sim S^{-\tau}$ of the avalanche size $S$, for sizes $S$ between small- and large-scale cutoffs $S_{\min } \ll S \ll S_{m}$. Pinned elastic manifolds are an important prototype for a much wider class of phenomena, exceeding physics and reaching for example economy and finance, where extreme (and sometimes catastrophic) events are sufficiently frequent and large to dominate most 
observables. In this context, it is clearly of importance to understand how the avalanche-size probability is cutted off at large scales, $S>S_{m}$.

Although avalanche motion of pinned manifolds has been studied for a while $[32,33,34]$, most work was focused on measuring the avalanche-size exponent $\tau$. A scaling argument proposed by Narayan and Fisher [35] on the assumption that the avalanche density remains finite at the depinning threshold, relates the exponent $\tau$ with the roughness exponent $\zeta$ via:

$$
\tau=2-\frac{2}{d+\zeta}
$$

Here $\zeta$ is the roughness exponent at the depinning transition. On the other hand the cut-off of large avalanches depend either on finite size effects either on the value of the external force. In the latter case scaling arguments give:

$$
S_{m} \sim L_{\text {relax }}^{d+\zeta} \sim\left|f-f_{c}\right|^{-\frac{d+\zeta}{2-\zeta}} .
$$

To describe the full statistics of avalanches we have to specify how the dynamics is implemented for a system of finite size $L$. An intuitive protocol, used in experiments of Barkausen noise, is shown in Fig. 8 where the interface is prepared in a metastable state at force $f=0$ and force is slowly increased up to the critical value $f_{c}$. Avalanches obtained using this method are not identically distributed because the cut-off $S_{m}$ depends on the distance to the critical point which is not constant during the evolution.

In our works we have replaced the external force $f$ with a an external parabolic potential to provide a proper definition of the steady state avalanche dynamics. To be more precise, the zero-temperature Langevin dynamics is described by the equation of motion:

$$
\begin{aligned}
\partial_{t} u(x, t) & =\mathcal{F}_{w(t)}(x, u(x, t)) \\
\mathcal{F}_{w}(x, u(x)) & =m^{2}(w-u(x))+c \nabla^{2} u(x)+F(x, u(x)),
\end{aligned}
$$

where $\mathcal{F}_{w}(x, u)$ is the total force acting on the manifold, $c$ is the elastic constant and $F(x, u)$ is the random pinning force. The parabolic potential is centred in $w$ and its curvature, $m^{2}$, acts as a mass for the field 
$u$. For random field (RF) disorder, $F(x, u)$ is short-ranged with correlations $\overline{F(x, 0) F\left(x^{\prime}, u\right)}=\Delta_{0}(u) \delta^{d}\left(x-x^{\prime}\right)$. For random bond (RB) disorder this force is derived from a short-ranged random potential $V(x, u)$, $F(x, u)=-\partial_{u} V(x, u)$.

Starting from an arbitrary initial condition $u_{\text {init }}(x)$, and giving $w=$ $w_{0}$, the manifold moves to a locally stable state $u_{w_{0}}(x)$, i.e., a zero-force state $\mathcal{F}_{w_{0}}\left(x, u_{w_{0}}(x)\right)=0$ which is stable to small deformations. Increasing $w, u(x)$ increases slightly, while the configuration remains stable. At some $w=w_{1}$, the state becomes unstable and the manifold moves until it is blocked again in a new locally stable state $u_{w_{1}}(x)$. We are interested in the centre of mass displacement

$$
u(w)=L^{-d} \int d x u_{w}(x) .
$$

The function $u(w)$ exhibits jumps at discrete values of $w$ and is in general dependent on the initial condition. However, due to the no-passing rule [36], we can prove that there exists a $w^{*}>w_{0}$ such that the orbit $u_{w>w^{*}}(x)$ becomes independent of the initial condition $u_{\text {init }}(x)$, and $w_{0}$. A stationary state is thus reached after a finite $w-w_{0}$, on which we focus. In Fig. 9 we show how the algorithm works, for $d w$ very small we are able to isolate a single avalanche event.

\subsection{Critical force and autocorrelation function}

It is useful to introduce the process [37]:

$$
x(w)=m^{2}[w-u(w)] .
$$

For a given location of the parabolic well, $x(w)$ represents the average force on the elastic interface in presence of a parabolic well. This force should is compensated by the presence of the impurity which pinned the system. The critical force for this system is thus given by:

$$
f_{c}(m)=\overline{x(w)}=m^{2} \overline{[w-u(w)]}
$$

when $m \rightarrow 0$ we have $f_{c}(m) \rightarrow f_{c}\left(f_{c}(m)\right.$ is independent of $L$ if $L m \gg 1)$. In Fig. 10 we show a typical realization of the process $x(w)$. 

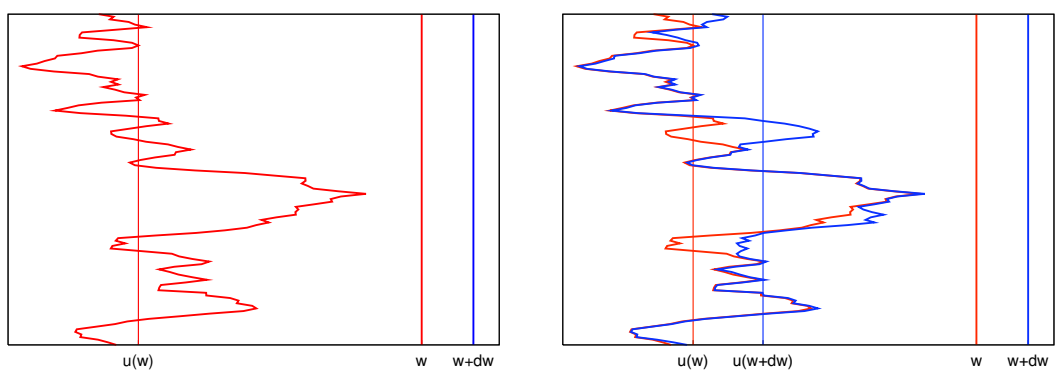

Fig. 9 - For a given $w$ the manifold moves to a metastable state $u_{w}(x)$, i.e. a state dynamically stable to infinitesimally small deformations. Then one increases $w$, and a smooth forward deformation of $u_{w}(x)$ results (for smooth short-scale disorder) while the state remains stable. At some $w=w_{1}$ the state becomes unstable and the manifold, for $w=w_{1}^{+}$ moves until it is blocked again in a new metastable state $u_{w_{1}}(x)$ (also locally stable). This process is called an avalanche and the centre of mass $u(w)$ bas a jump located at $w=w_{1}$.

We see that, due to the presence of avalanches, this process display vertical jumps and fluctuation around the mean value. The statistical tilt symmetry assures that the quadratic part of the hamiltonian (and thus the mass $m$ ) is not renormalized. This means that the length associated to the bare mass $L_{m}=1 / m$ fixes the distance from the critical point (located at $f_{c}$ ) as $m^{1 / \nu}=m^{2-\zeta}$. The finite size effects on the force takes then the form:

$$
f_{c}(m)=f_{c}+c_{1} m^{2-\zeta}+\ldots
$$

The fluctuations around this value depend on $L$ and $m$. In the limt $L \gg L_{m}=1 / m$, the interface can be modelled as a collection of independent interfaces of size $L_{m}$ and the central limit theorem assures that fluctuations should decrease as $\sqrt{L_{m} / L}$. On the other hand using that the distance from the critical point is $m^{1 / \nu}=m^{2-\zeta}$ and that $w$ scales as $L_{m}^{\zeta}$ we can write

$$
x(w)=f_{c}(m)+\frac{m^{1 / \nu}}{\sqrt{L / L_{m}}} y\left(w / L_{m}^{\zeta}\right)=f_{c}(m)+\frac{m^{3 / 2-\zeta}}{\sqrt{L}} y\left(w m^{\zeta}\right),
$$



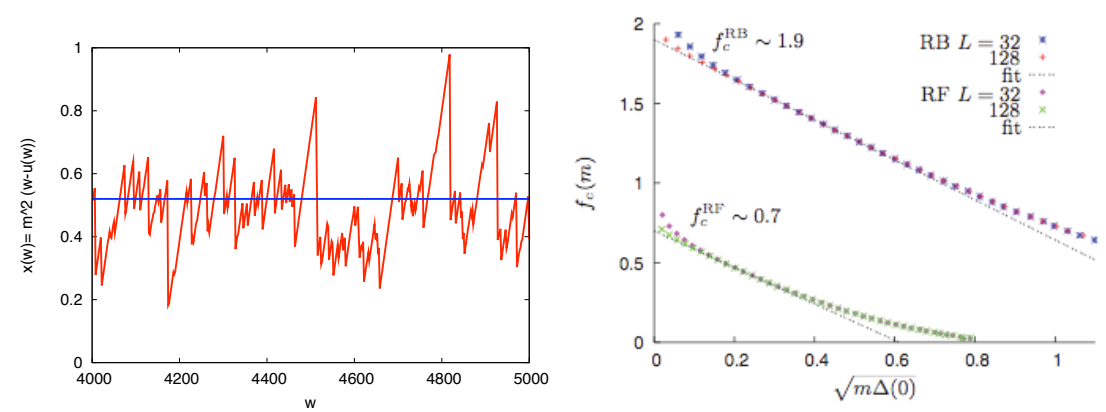

Fig. 10 - Left: Typical realization of the process $x(w)$. Right: Critical force, finite size effect study.

where the rescaled process $y(z)$ is independent of $m$ and $L$ as $m \rightarrow 0$ and $L m \gg 1$. Approaching the critical point $y(z)$ displays universal behavior and becomes independent of most microscopic details. Using the set up with the parabolic well we can measure the autocorrelation function of $x(w)$ :

$$
\begin{aligned}
& \left.m^{4} \overline{[w-u(w)]\left[w^{\prime}-u\left(w^{\prime}\right)\right.}\right]^{c}=L^{-d} \Delta_{m}\left(w-w^{\prime}\right) . \\
& \Delta(w)=m^{3-2 \zeta} \tilde{\Delta}\left(w m^{\zeta}\right)
\end{aligned}
$$

where $\Delta_{m}(w) \rightarrow \Delta(w)$ as $m \rightarrow 0$ and $\tilde{\Delta}(z)$ is the autocorrelation function of the process $y(z)$.

We have studied the behavior of the critical force $f_{c}(m)$ for two classes of disorder the RF and the RB. From (14) one has $\sqrt{\Delta(0) m} \sim$ $\mathrm{m}^{2-\zeta}$, yielding a parameter-free linear scaling shown in Fig. 10 [37]. For large $m$ the scaling is non-linear, while for smaller $m$ it is linear up to the scale where the correlation length becomes of the order of $L(m L \approx 5)$. The critical force of the infinite system is defined here in an unambiguous way, as $f_{c}=f_{c}(m=0)$. One can see on Fig. 10 that the slope, $c_{1}$, of the two curves coincides. Indeed, $c_{1}$ is a universal amplitude, depending on microscopic details only through the renormalized elastic coefficient $c$; here $c \approx 1$ for both models of Fig. 10 . 


\subsection{Avalanche statistics}

We can now study in detail the avalanche statistics [38]. The size $S$ of an avalanche is defined as the area swept by the line as it jumps between the two consecutive metastable states. The distribution of avalanche sizes is expected to exhibit universality, i.e. independence of short scale details, for sizes $S>S_{\min }$. The short-scale cutoff $S_{\min }$ corresponds to the area spanned by a single monomer on the scale of the discretization of the disorder (in our units $S_{\min } \simeq 1$ ). We define the (normalized) distribution of avalanche sizes $P(S)$, as well as its moments

$$
\left\langle S^{n}\right\rangle=\frac{1}{N} \sum_{i=1}^{N} S_{i}^{n}=\int_{0}^{\infty} d S S^{n} P(S)
$$

from the sequence of measured avalanches $S_{i}, i=1, \ldots, N$.

In the limit $m=0$ a critical point is reached, resulting in a powerlaw distribution of avalanche sizes. To properly define the problem, including the stationary measure, it is essential to consider a small $m>0$. Then, the correlation length $L_{m}=1 / m$ is large in the small- $m$ regime considered here. As a result, the distribution of avalanche sizes is cut-off by the large scale $S_{m} \gg S_{\min }$. Using the setting with a parabolic well the large scale cut off is expected to scale as $S_{m} \sim L_{m}^{d+\zeta} \sim m^{-d-\zeta}$ at small $m$. It is useful to express $S_{m}$ in terms of the moments $\left\langle S^{n}\right\rangle$. The definition depends on the value of $\tau$, the critical exponent of avalanche size statistics.

- For $\tau<1 S_{\min } \rightarrow 0,\langle S\rangle \sim S_{m}$

- For $\tau>1 S_{\min }$ must be finite in order to have finite moments. In particular the moments write $\left\langle S^{n}\right\rangle \sim S_{m}^{n+1-\tau} S_{\text {min }}^{\tau-1}$, so that we can define

$$
S_{m}:=\frac{\left\langle S^{2}\right\rangle}{2\langle S\rangle} .
$$

The scale $S_{m}$ is important as it allows to define universal functions. In the variable $s:=S / S_{m}$ avalanche statistics becomes universal. Nevertheless, if the exponent $\tau$ satisfies $2>\tau>1$, which is the case here, 
then the distribution of avalanche should always depend on $S_{\min }$ which assures a proper normalization.

A fully universal quantity is the function $p(s)$, defined via the relation:

$$
P(S) d S=\frac{\langle S\rangle}{S_{m}} p\left(\frac{S}{S_{m}}\right) \frac{d S}{S_{m}} .
$$

The function $p(s)$ is universal and depends only on the space dimension $d$. Note that the normalized probability $P(S)$ depends on the cut-off $S_{\min }$ via the first moment $\langle S\rangle$ which cannot be predicted by the theory, hence is an input from the numerics. It is important to stress that while the function $p(s)$ is universal and convenient for data analysis, it is not a probability distribution and it is not normalized to unity. Rather, it satisfies from its definition (17) and using (16), the two normalization conditions

$$
\begin{aligned}
\langle s\rangle_{p} & =\int d s s p(s)=1 \\
\left\langle s^{2}\right\rangle_{p} & =\int d s s^{2} p(s)=2 .
\end{aligned}
$$

A practical algorithm to compute $p(s)$ from a sequence $S_{1}, S_{2}, \ldots$ of avalanches is defined as:

- compute $\langle S\rangle$ and $\left\langle S^{2}\right\rangle$

- compute $S_{m}=\frac{\left\langle S^{2}\right\rangle}{2\langle S\rangle}$

- compute $s_{1}=S_{1} / S_{m}, S_{2} / S_{m}, \ldots s_{n}=S_{n} / S_{m}$

- make the histogram of the $s_{i}$

- multiply the heights of the histograms by $\frac{\left\langle S^{2}\right\rangle}{2\langle S\rangle^{2}}=\frac{S_{m}}{\langle S\rangle}$

The rescaled avalanche-size distribution can be written as

$$
p(s)=s^{-\tau} f(s)
$$




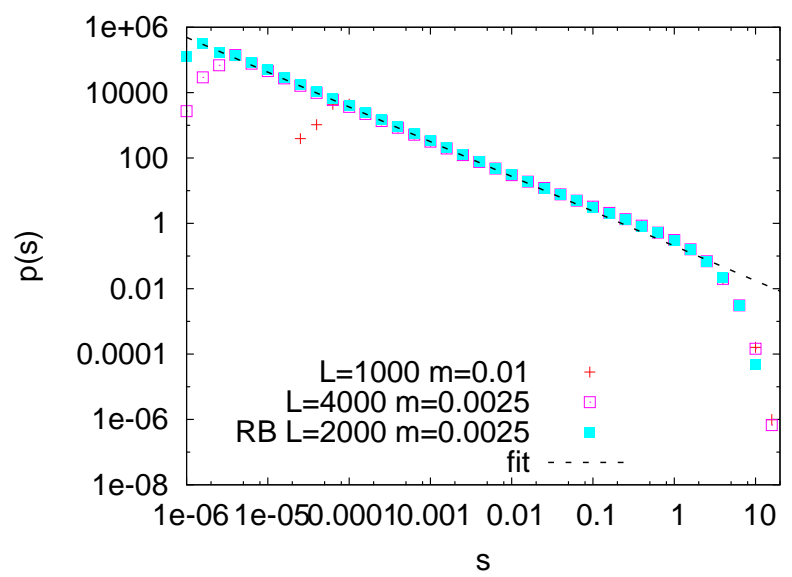

Fig. 11 - The function $p(s)$ for $R F$ and $R B$ disorder $(d=1)$. A fit with a power law gives the exponent $\tau=1.08 \pm 0.02$. The agreement with Eq. (6) is discussed in the text.

where $\tau$ is the avalanche-size exponent, and $f(s)$ the universal cut-off function $^{1}$, which tends to a constant for $s \rightarrow 0$.

For the present model, the only analytical prediction concerns the exponent $\tau$, via the above mentioned conjecture [35] of Eq.(6). For $d=$ 1 , a direct power-law fit of our numerical data gives

$$
\tau_{\text {num }}^{d=1}=1.08 \pm 0.02 .
$$

This value has to be compared with the conjecture of Eq. (6). The roughness exponent is known numerically with a good accuracy from system sizes $\left(L \sim 10^{3}\right)$ and $m=0$, as $\zeta=1.26 \pm 0.01$ [17]. This value for $\zeta$ gives $\tau_{\text {conj }}=1.115 \pm 0.005$. Hence the estimate (21) is slightly smaller, but still consistent with the value of $\tau$ obtained from the conjecture.

Exact solution of a mean field toy model of avalanches allows to compute the full universal scaling function:

$$
f_{\mathrm{MF}}(s)=\frac{1}{2 \sqrt{\pi}} e^{-s / 4} .
$$

\footnotetext{
${ }^{1}$ By universal we mean w.r.t. short scale details. Of course this function is characteristic of a large scale cut-off provided by a parabolic well.
} 

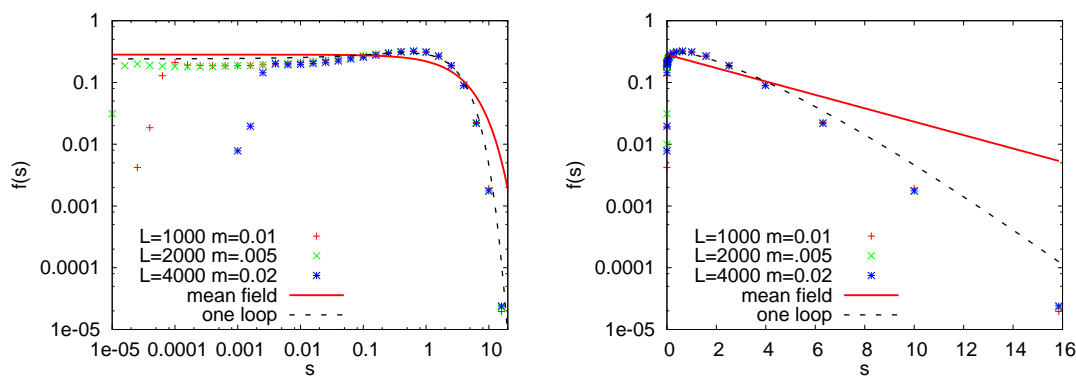

Fig. 12 - Function $f(s)$ for RF disorder $(d=1)$. The red solid curve is given by Eq.(22), the black dashed line by Eq.(23), with $A=0.852, B=1.56$ and $C=0.56$. Left: Blow up of the power-law region. Right: Blow up of the tail region.

which is expected to be exact above the upper critical dimension $d=$ $d_{\mathrm{uc}}=4$. The Functional Renormalization Group calculation gives a finite dimension correction to the mean field result, at the first order in the $\varepsilon=4-d$ expansion we have the following prediction [39]

$$
f(s)=\frac{A}{2 \sqrt{\pi}} \exp \left(C \sqrt{s}-\frac{B}{4} s^{\delta}\right),
$$

with exponents

$$
\begin{aligned}
& \tau=\frac{3}{2}+\frac{3}{8} \alpha=\frac{3}{2}-\frac{1}{8}\left(1-\zeta_{1}\right) \varepsilon \\
& \delta=1-\frac{\alpha}{4}=1+\frac{1}{12}\left(1-\zeta_{1}\right) \varepsilon
\end{aligned}
$$

where $\alpha=-\frac{1}{3}\left(1-\zeta_{1}\right) \varepsilon$ and $\zeta_{1}=1 / 3$ for the RF class, relevant to the present study. The constants $A, B$ and $C$ depend on $\varepsilon$, and must satisfy the normalization conditions (18), (19). At first order in $\varepsilon$ they are $C=$ $-\frac{1}{2} \sqrt{\pi} \alpha, B=1-\alpha\left(1+\frac{\gamma_{\mathrm{E}}}{4}\right), A=1+\frac{1}{8}\left(2-3 \gamma_{\mathrm{E}}\right) \alpha, \gamma_{\mathrm{E}}=0.577216$. As usual, the one-loop results for the exponents $\tau, \delta$ and for the parameters $A, B$, and $C$ are exact up to $O\left(\varepsilon^{2}\right)$.

Our numerical data for the avalanche-size distribution $p(s)$ are plotted in Fig. 12, with emphasis either on the power-law region or on the tail. Note that for the different values of $m$ and $L$ used here, the 
data have converged, with the exception of the last point for very large avalanches (over-suppressed by the finite size of the interface in the smallest samples), and the region of very small avalanches (which are cut off at $\left.s \approx 1 / S_{m}\right)$.

\section{CONCLUSIONS}

In Conclusion we have discussed the results on two aspects of the dynamics of an elastic interface in random medium: the activated dynamics or creep, and the avalanche dynamics or depinning.

Despite its relevance for experiments and the theoretical efforts made, the description of the creep regime remains a very challenging problem. Most of the few predictions proposed in litterature are based on strong assumptions that should be verified, at least numerically. In particular it should be shown clearly that a small drive does not change the short scale properties of the interface that remains locally in equilibrium even when it is driven in an out of equilibrium steady state. Verifications are needed not only for the assumptions of creep theory, but for the predictions too. It is true that the main prediction, i.e. the creep formula for the velocity, has received an important confirmation with the experiment of magnetic domain wall [1], but on one side this is the unique experiment that clearly shows a creep regime and on the other side numerical simulations are still far to be conclusive. New algorithms have been developed to capture the secrets hidden in the deep metastable states of the disorder landscape. We are actively working in this direction.

The depinning transition has been much more understood, but different directions are open for future researches. Let me mention some directions of my present research activity:

- The description of the universal behavior is of a little help for the estimation of the threshold above which the interface moves even at zero temperature. In fracture the estimation of this threshold should give information about the toughness of the material [40]. This should be true at least for fragile materials for which plasticity plays a minor role. If this is true, to understand how the threshold behaves as a function of the impurity concentration, the impurity 
size and the elastic moduli of the material, will allow to control the toughness of a family of materials. This is a major task in fracture theory.

- In a real system, the sequence of avalanches as a function of time display strong correlation in time. In particular the phenomenological Omori law, verified for the seismic activity, predicts power law correlations. Unfortunately an elastic interface in the steady state regime shows a quasi-Poissonian statistics with absence of correlations, both in time and in intensity. Which is the missing ingredient in the model? Recently, a model reproducing aftershock statistics has been proposed [29]. This model incorporates an internal degree of freedom which relax the contact between the disordered system and the elastic interface. We studied a simple model of viscoelastic interface that reproduce a large part of the observations of the earthquakes statistics [41]

- Collective re-organizations are also observed in the dynamics of amorphous solids under shear deformation [42, 43]. Here, when a site slips, it triggers a shock wave which in turn can either stabilize or destabilize other sites. This is at variance with what is observed in the avalanches discussed so far, where an unstable site can only destabilize other sites. We have recently introduced a scaling description of the yielding transition in term of universal exponents [44].

\section{AKNOWLEDGEMENTS}

We acknowledge very fruitful collaborations with T. Giamarchi, A. Kolton, P. Le Doussal, W. Krauth and K. Wiese. 


\section{REFERENCES}

[1] S. Lemerle et al., Phys. Rev. Lett. 80, 849 (1998).

[2] V. Repain et al., Europhys. Lett. 68, 460 (2004).

[3] S. Moulinet, C. Guthmann, and E. Rolley, Eur. Phys. J. E 8, 437 (2002).

[4] E. Bouchaud, J.-P. Bouchaud, D.S. Fisher, S. Ramanathan, J.R. Rice, Journal of the Mechanics and Physics of Solids 50, 1703 (2002).

[5] D. Bonamy, E. Bouchaud, Physics Reports 498, 1-44 (2011).

[6] L. Ponson, Phys. Rev. Lett. 103, 055501 (2009).

[7] G. Blatter et al., Rev. Mod. Phys. 66, 1125 (1994).

[8] G. Grüner, Rev. Mod. Phys. 60, 1129 (1988).

[9] A. I. Larkin and Y. N. Ovchinnikov, J. Low Temp. Phys 34, 409 (1979).

[10] P. W. Anderson and Y. B. Kim, Rev. Mod. Phys. 36, 39 (1964).

[11] L. B. Ioffe and V. M. Vinokur, J. Phys. C 20, 6149 (1987).

[12] P. Chauve, T. Giamarchi, and P. Le Doussal, Phys. Rev. B 62, 6241 (2000).

[13] A. Rosso, W. Krauth, Phys. Rev. E 65, 025101(R) (2002).

[14] D. S. Fisher, Phys. Rev. B 31, 1396 (1985).

[15] T. Nattermann, S. Stepanow, L. H. Tang, and H. Leschhorn, J. Phys. (Paris) 2, 1483 (1992).

[16] O. Narayan and D. Fisher, Phys. Rev. B 48, 7030 (1993).

[17] A. Rosso, A. K. Hartmann, and W. Krauth, Phys. Rev. E 67, 021602 (2003). 
[18] A. B. Kolton, A. Rosso, T. Giamarchi, W. Krauth, Physical Review Letters 97, 057001 (2006).

[19] A. B. Kolton, A. Rosso, T. Giamarchi, W. Krauth, Phys. Rev. B 79, 184207 (2009).

[20] U. Schulz, J. Villain, E. Brézin, and H. Orland, J. Stat. Phys. 51, 1 (1988).

[21] B. Drossel, M. Kardar, Phys. Rev. E 52, 4841 (1995).

[22] O. Duemmer, W. Krauth, Phys. Rev. E 71, 61601 (2005).

[23] A. B. Kolton, A. Rosso, T. Giamarchi, Phys. Rev. Lett. 94, 047002 (2005).

[24] J. S. Urbach, R. C. Madison and J. T. Markert, Phys. Rev. Lett. 75 (1995) 276-279.

[25] JP. Sethna, KA. Dahmen and CR. Myers, Nature 410 (2001) 242250.

[26] P. Moretti, M.C. Miguel, M. Zaiser and S. Zapperi, Phys. Rev. B 69 (2004) 214103.

[27] D. Bonamy, S. Santucci and L. Ponson, Phys. Rev. Lett. 101 (2008) 045501.

[28] D.S. Fisher, K. Dahmen, S. Ramanathan and Y. Ben-Zion, Phys. Rev. Lett. 78 (1997) 4885-4888.

[29] E. A. Jagla and A. B. Kolton, arXiv: 0901.1907 (2009).

[30] C. Tang and P. Bak, Journal Of Statistical Physics 51 (1988) 797802.

[31] D. Dhar, Physica A 369 (2006) 29-70.

[32] O. Narayan and AA. Middleton, Phys. Rev. B 49 (1994) 244-256.

[33] F. Lacombe, S. Zapperi and H.J. Herrmann, Phys. Rev. B 63 (2001) 104104 (7 pages). 
[34] A. Tanguy, M. Gounelle and S. Roux, Phys. Rev. E 58 (1998) 157790.

[35] O. Narayan and D.S. Fisher, Phys. Rev. B 48 (1993) 7030-42.

[36] A. A. Middleton, Phys. Rev. Lett. 68, 671 (1992).

[37] A. Rosso, P. Le Doussal and K.J. Wiese, Phys. Rev. B 75 (2007) 220201, cond-mat/ 0610821.

[38] A. Rosso, P.Le Doussal, K.J. Wiese, Phys. Rev. B 80, 144204 (2009).

[39] P. Le Doussal and K.J. Wiese, arXiv: 0812.1893 (2008).

[40] V. Demery, L. Ponson, A. Rosso, EPL, 105, 34003, (2014).

[41] E. A. Jagla, F. Landes, A. Rosso, Phys Rev Lett. 174301 (2014).

[42] P. Moretti, B. Cerruti, M.C. Miguel, PLoS One 6 (6), e20418 (2011).

[43] M. Talamali, V. PetŠjšs, D. Vandembroucq, S. Roux, PRE 84 016115 (2011).

[44] J. Lin, E. Lerner, A. Rosso and M. Wyart, PNAS (2014) 